\title{
UM MODELO PARA A ANÁLISE DO BALANÇO E EFICIÊNCIA ENERGÉTICA NA LOGÍSTICA DE TRANSPORTE DUTOVIÁRIO PARA EXPORTAÇÃO DO ETANOL
}

\author{
Fábio César Bovolenta ${ }^{1}$ \& Marco Antônio Martin Biaggioni ${ }^{2}$ \\ RESUMO: O presente trabalho analisou o fluxo energético considerando uma rota atualmente projetada para o \\ escoamento de etanol da região Centro-Oeste do Brasil para exportação, mais precisamente, do município de Aparecida \\ do Taboado (MS) até o porto de São Sebastião (SP). A rota estudada, com $837 \mathrm{~km}$ percorridos, teve um único modal \\ combinado em dois trechos, duto-dutoviário. Foram utilizados fatores energéticos, diretos e indiretos, envolvidos nas \\ operações para contabilizar os inputs e outputs do sistema, tais como: óleo diesel, lubrificantes, graxas, consumo de \\ energia indireta de máquinas e equipamentos, o consumo de energia de mão de obra, o consumo de energia elétrica e o \\ consumo de energia na depreciação e manutenção das vias, considerados no modelo proposto. O resultado obtido desta \\ rota foi um consumo específico de energia de $0,14 \mathrm{MJ}$ km-1 m-3. Quando contabilizados o Ganho Energético líquido \\ (GEl), a Eficiência Energética global (EEg) e o Balanço Energético renovável (BEr), que foram os indicadores \\ energéticos adotados, obteve-se respectivamente: 1.585.958.977,00 MJ; 200,72 e 1.593.900.000,00 MJ.
}

PALAVRAS-CHAVE: Exportação, etanol, logística, transportes dutoviário, unimodalidade, análise energética, eficiência energética, balanço energético, indicadores energéticos.

\section{A Model For The Analysis Of Energy Balance And Efficiency In Duct Logistics Transportation Of Etanol Exportation}

\begin{abstract}
This paper analyzed the energy flow of a route currently designed to transport ethanol from the Midwest region of Brazil for exportation, more precisely from the city of Aparecida do Taboado (MS) to the port of São Sebastiao (SP). The route studied a single modal combined into two pieces, duct - duct. The direct and indirect energy, involved in the operations were used to account for the inputs and outputs of energy from and into the system. The energy input and output were the variables, diesel fuel, lubricants, greases, indirect energy consumption of machinery and equipment, power consumption of labor, the energy consumption and energy consumption in depreciation and maintenance of roads. We found that this route has specific energy consumption of $0,14 \mathrm{MJ} \mathrm{km}-1 \mathrm{~m}-3$. The Net Energy Gain (GEl), the Energy Efficiency global (EEg) and Renewable Energy Balance (BEr), which were the energy indicators adopted in this study were obtained respectively: 1.585.958.977,00 MJ; 200,72 and 1.593.900.000,00MJ.
\end{abstract}

KEYWORDS: Exports, ethanol, logistics, duct transportation, unimodality, energy analysis, energy efficiency, energy balance, energy indicators.

\section{INTRODUÇÃO}

Segundo a ÚNICA - União da Indústria da Cana-deAçucar (2012), a cana de açúcar ocupa cerca de 7 milhões de hectares ou $2 \%$ de toda a terra arável do Brasil que é o maior produtor mundial, seguido por Índia, Tailândia e Austrália. As regiões de cultivo são Sudeste, Centro-Oeste, Sul e Nordeste, permitindo duas safras por ano e a produção de açúcar e etanol para os mercados interno e externo, durante todo o ano.

\footnotetext{
${ }^{1}$ Prof., Departamento de Navegação e Logística, FATEC-Jahu. E-mail: fabiobovolenta@ig.com.br

${ }^{2}$ Prof., Departamento de Engenharia Rural, FCA/UNESP-Botucatu. Email: biaggioni@fca.unesp.br
}

Para a CONAB - Companhia Nacional de Abastecimento (2012), a produção estimada de cana esmagada em 2012 foi de, aproximadamente, 300,6 milhões de toneladas, gerando 23,7 bilhões de litros de etanol, $14,17 \%$ menor que a produção da safra 2010/11. A queda foi devido a fatores climáticos aqui no Brasil e mercado internacional do açúcar e do etanol que oscilou. Deste total, 9,1 bilhões de litros foram de etanol anidro e 14,6 bilhões de litros de etanol hidratado. Por estes números, o etanol anidro teve um aumento de $13,97 \%$ na produção e o etanol hidratado teve uma redução de $25,69 \%$, quando comparados com a produção de etanol da safra anterior. 
Para CEISE - Centro Nacional das Indústrias do Setor Sucroenergético e Biocombustíveis (2012), os grupos sucroalcooleiros instalados no Brasil estão otimistas com as oportunidades no mercado de etanol. Na safra 2011/12, as exportações de etanol atingiram 1,64 bilhão de litros e a importação 1,66 bilhão de litros, com previsão de crescimento para a safra 2012/13.

Para o escoamento deste etanol até os portos exportadores, as empresas e o governo brasileiro buscam sempre a diminuição do chamado "custo Brasil". Esse que é composto por impostos de movimentação e armazenagem de materiais; custos ligados a dutovias; custos de administração, controle, organização e manuseio de estoques; custos embutidos em armazéns, pátios, tanques, silos e almoxarifados; custos de transporte dutoviário; custos presentes em sistemas portuários; e custos relacionados a encargos de mão de obra logística.

A utilização dos princípios logísticos tem sido muito valorizada na movimentação e armazenagem de materiais. Ela visa, principalmente, a otimização da eficiência das operações de transportes.

Cada modalidade de transporte oferece uma série de vantagens e desvantagens na movimentação de cargas líquidas. O transporte dutoviário é caracterizado pela movimentação de cargas com alto peso específico, fretes menores, mas somente viável em médias e longas distâncias.

A LOGUM Logística S.A. é a empresa responsável pela construção e operação do sistema logístico de etanol (logística, carga, descarga, movimentação e estocagem, operação de portos e terminais terrestres e aquaviários) que envolverá transportes multimodais: dutos, hidrovias (barcaças), rodovias (caminhões-tanques) e cabotagem (navios) em conjunto. Ela é o resultado da integração dos projetos de logística de etanol da UNIDUTO (conglomerado formado por 12 grandes grupos do setor sucroalcooleiro do Brasil, que operam 81 usinas), Centro Sul Transportes e Logística e PMCC (grupo Petrobras, Mitsui e Camargo Corrêa).

Tendo como base o projeto de escoamento de etanol da região centro-oeste do país projetada pela Logum Logística S.A., tomar-se-á, como ponto de origem de um estudo de análise energética multimodal de transporte, o município de Aparecida do Taboado (SP) e, como destino, o porto de São Sebastião (SP).

Bovolenta e Biaggioni (2009), descreveram em seu artigo, que a energia consumida e produzida num sistema logístico depende de alguns fatores diretos e indiretos de operação. O óleo diesel, por exemplo, apresenta acentuada demanda de consumo energético em rodovias, ferrovias e hidrovias. Outros fatores de consumo energético também existem, tais como, de máquinas e equipamentos, consumo de energia de mão de obra, consumo de energia elétrica e o consumo de energia para manutenção da via.

Tendo em vista as dutovias representarem uma alternativa com grande potencial inovador quando se busca uma logística de transporte mais sustentável, mais precisamente no que se refere ao óleo diesel, o presente trabalho objetiva realizar um modelo para a análise do balanço e eficiência energética de escoamento do etanol, da região Centro Oeste do Brasil para um porto marítimo exportador, contemplando a unimodalidade de transporte, ou seja, o modal dutoviário.

\section{MATERIAL E MÉTODOS}

O estudo iniciou-se pela roteirização do etanol, citado abaixo no item 2.1. Logo após, foi realizado a delimitação dos materiais e equipamentos envolvidos na movimentação do etanol, citados no item 2.2. A conversão dos fatores físicos em energéticos e os cálculos dos indicadores energéticos são citados nos itens 2.3 e 2.4 .

O fator de equivalência de carga (F), citado no item 2.1, é um número utilizado para realizar a equiparação entre as cargas transportadas em cada trecho de uma determinada rota, uma vez que, em cada trecho temos um modal diferente que apresenta um volume diferente de etanol transportado. Assim, o fator de equivalência igual a 1 (carga de referência) prevaleceu sempre no trecho onde o modal transportou o maior volume de etanol.

Ao analisar o transporte para exportação de etanol (Figura 1), adotou-se como ponto de origem, o município de Aparecida do Taboado (MS). A escolha deste foi para possibilitar a análise em um local que seria uma grande base coletora de etanol da região CentroOeste do país com capacidade de armazenagem de 20 mil m3 de etanol e capacidade de fluxo anual de 2,4 milhões de $\mathrm{m} 3$ de etanol. Como ponto intermediário de transbordo, adotou-se o município de Paulínia (SP), por ser uma das principais bases distribuidoras de etanol do estado de São Paulo com capacidade de armazenagem de 224 mil m3 de etanol e capacidade de fluxo anual de 8,9 milhões de $\mathrm{m} 3$ de etanol. Como ponto de destino para a exportação do etanol, adotou-se o município de São Sebastião (SP), litoral norte paulista, por ter um porto marítimo já estruturado para a logística de etanol. A capacidade de armazenagem do porto de São Sebastião é de 260 mil m3 de etanol e capacidade de fluxo anual de 12,9 milhões de $\mathrm{m} 3$ de etanol. 


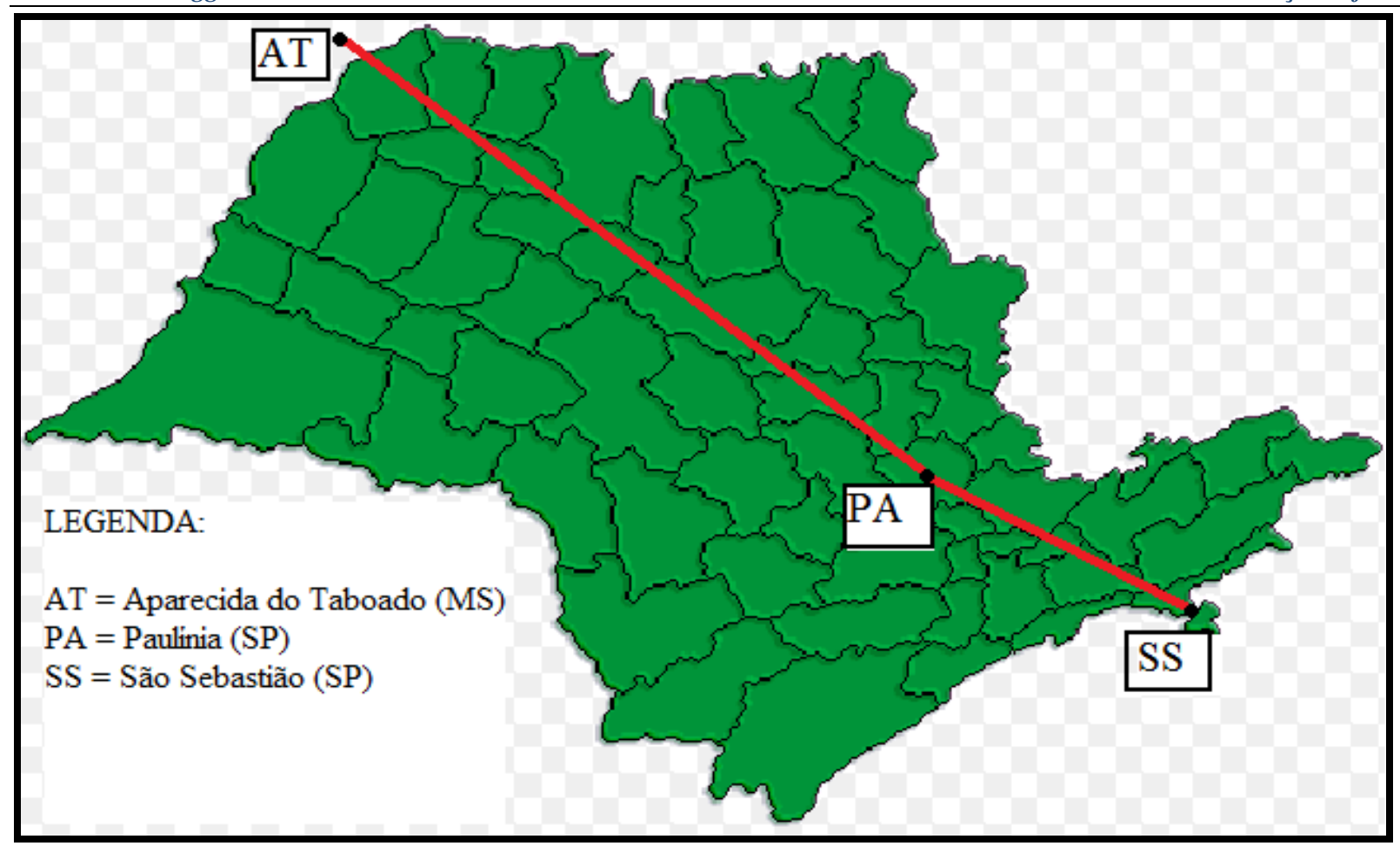

Figura 01 - Mapa ilustrativo do escoamento do etanol, de Aparecida do Taboado (MS) até o porto de São Sebastião (SP) por dutovia. Fonte: O autor.

\subsection{Roteirização}

Neste modelo logístico de análise de transporte é utilizado o unimodal com dois trechos, composto pelo dutoviário. Tem como origem a cidade de Aparecida do Taboado (MS) e percorre $837 \mathrm{~km}$ até o porto marítimo de São Sebastião (SP).

No primeiro trecho, seguiriam em alcoodutos $700 \mathrm{~m}^{3} \mathrm{~h}^{-1}$ de etanol até os tanques de estocagem na cidade de Paulínia (SP) totalizando parcialmente $597 \mathrm{~km}$ de distância em, aproximadamente, 99 horas. No segundo e último trecho, o etanol seguiria novamente $\mathrm{em}$ alcoodutos a $700 \mathrm{~m}^{3} \mathrm{~h}^{-1}$ até os tanques de estocagem no porto marítimo da cidade de São Sebastião (SP) num total parcial de $240 \mathrm{~km} \mathrm{em}$, aproximadamente, 40 horas.

O "fator de equivalência" de carga é de "1,0" (carga de referência) para o transporte dutoviário no $1^{\circ}$ trecho e " $2,48^{\prime}$ " para o transporte dutoviário no $2^{\circ}$ trecho.

\subsection{Delimitação dos materiais e equipamentos}

O transporte de etanol seria realizado por tubulações de aço com diâmetro médio de 24 polegadas. A massa de cada duto com 18 metros de comprimento é de $8.000 \mathrm{~kg}$ cada (ANP, 2010) e vida útil em anos de uso de 40 anos (CERNE, 2000). Ao longo de todo o sistema de transporte e movimentação do etanol por tubulações haveriam skids de descarga (equipamentos compostos de mangueiras para acoplamento nos caminhões, tanque de desaeramento, bomba de transferência, instrumentos de medição de densidade e volume) que descarregam dos caminhões e transportam os produtos até seu respectivo tanque de armazenamento/estocagem (ANP, 2010).
Dos tanques de armazenamento/estocagem, o etanol seria movimentado através das bombas intermediárias Booster B-7776 até a sucção das bombas principais Booster B-35001 nos dutos principais de $15 \mathrm{HP}$ e destas seria bombeado até a REPLAN em Paulínia (SP) ou ao porto de São Sebastião (SP). Haveria estações de bombeamento a cada $200 \mathrm{~km}$ se necessário (ANP, 2010).

As características das bombas centrífugas dos dutos, equipamentos estes que iriam transferir o etanol são: duas bombas com capacidade unitária de $700 \mathrm{~m}^{3} \mathrm{~h}^{-1}$, sendo que uma seria reserva. A vazão considerada no projeto foi de $3.000 .000 \mathrm{de} \mathrm{m}^{3} \mathrm{ano}^{-1}$ de etanol uma vez que, foi considerado no projeto que o mesmo operará, aproximadamente, $22 \mathrm{~h} \mathrm{dia}^{-1}$ (ANP, 2010).

\subsection{Conversão dos fatores físicos em energéticos}

A partir desse ponto converteu-se as diversas formas físicas encontradas em unidades energéticas (MJ). Assim sendo, converteu-se a energia das máquinas elétricas, quantificação da mão-de-obra utilizada, por operação, energia indireta de máquinas e equipamentos e energia indireta de depreciação de vias (BOVOLENTA, 2013).

\subsubsection{Energia do tipo direta de fonte industrial sob a forma elétrica:}

O conteúdo energético dos equipamentos elétricos utilizados no sistema (bombas hidráulicas booster) foram obtidos através da equação 1 (BOVOLENTA, 2013):

$$
E D I E=0,745 * 3,6 * P * T * F * N e
$$

Onde: 
EDIE = energia do tipo direta de fonte industrial sob a forma elétrica $(\mathrm{MJ})$;

$\mathrm{P}=$ potência $(\mathrm{HP})$;

$\mathrm{T}=$ tempo de utilização $(\mathrm{h})$;

$\mathrm{F}=$ fator de equivalência de carga;

$\mathrm{Ne}=$ número de equipamentos.

\subsubsection{Energia do tipo direta de fonte biológica sob a forma de mão de obra:}

A conversão do dispêndio energético devido a mão-deobra seguiu o trabalho descrito em (AINSWORTH, 1993). Eles classificaram o custo energético de atividades físicas humanas. É um esquema de codificação que classifica as atividades de acordo com seu propósito (por exemplo: esporte, ocupacional, automanutenção), tipo específico de atividade e sua intensidade enquanto taxa de trabalho metabólico em relação à taxa de trabalho metabólico - Metabolic Employment Tax (MET). O gasto energético corporal pode ser estimado para todas as atividades, atividades específicas ou tipos de atividades. Os valores de METs listados na Tabela 1 são para algumas atividades ocupacionais.

Tabela 01 - Dados para o cálculo do custo energético de atividades físicas humanas.

\begin{tabular}{lc}
\hline Atividades ocupacionais & MET \\
\hline Reforma de construções: & 5,5 \\
Trabalho de eletricista e bombeiro: & 3,5 \\
Bombeiro em geral (apagar incêndio): & 8,0 \\
Plantar com as mãos (florestas): & 6,0 \\
Operar equipamento pesado/automático: & 2,5 \\
Atividade policial: dirigir trânsito, de pé: & 2,5 \\
Atividade policial: dirigir uma viatura: & 2,0 \\
Atividade policial: fazer uma prisão: & 8,0 \\
Reparar calçados de forma geral: & 2,5 \\
Participar de reuniões em geral: & 1,5 \\
Trabalhos leves, na posição de pé: & 2,5 \\
Trabalhos moderado na posição de pé: & 3,0 \\
Trabalhos pesados na posição de pé: & 4,0 \\
Dirigir caminhão, carregar/descarregar: & 6,5 \\
Digitar computador: & 1,5 \\
Operar máquinas: & 2,6 \\
Controlador de qualidade: & 3,3 \\
Trabalhar em forno de tratamento térmico: & 3,4 \\
Operar prensas leves de forjamento: & 3,7 \\
Servente de pedreiro: & 5,0 \\
Pedreiros: & 5,2 \\
\hline Fonte: Ainswoth et al (1993). & \\
\hline
\end{tabular}

Fonte: Ainsworth et al. (1993).

A conversão foi feita através da Equação 2:

$E D F B=0,00418^{*} M E T * M * T * F * N P * N D$

Onde:

EDFB = energia do tipo direta de fonte biológica (MJ);

MET = taxa metabólica no repouso;

$\mathrm{M}=$ massa corporal $(\mathrm{kg})$;
$\mathrm{T}=$ tempo $(\mathrm{h})$;

$\mathrm{F}=$ fator de equivalência;

$\mathrm{NP}=$ número de pessoas;

$\mathrm{ND}=$ número de dias.

\subsubsection{Energia do tipo indireta de fonte industrial sob a forma de máquinas e equipamentos:}

A metodologia adotada para os cálculos da depreciação energética relativa às máquinas e equipamentos seguiu (MOREIRA, 2005), com adaptações. Os cálculos de energia indireta embutida foram baseados na massa multiplicada pelos seus respectivos coeficientes energéticos. Isto é multiplicado pelas horas de utilização e em função da vida útil. O acréscimo de $5 \%$ de "a" corresponde ao custo com reparos e $12 \%$ de "a $+b+c$ " corresponde ao custo com manutenção. Para determinação do consumo energético das máquinas e equipamentos foram adotadas as Equações 3, 4 e 5:

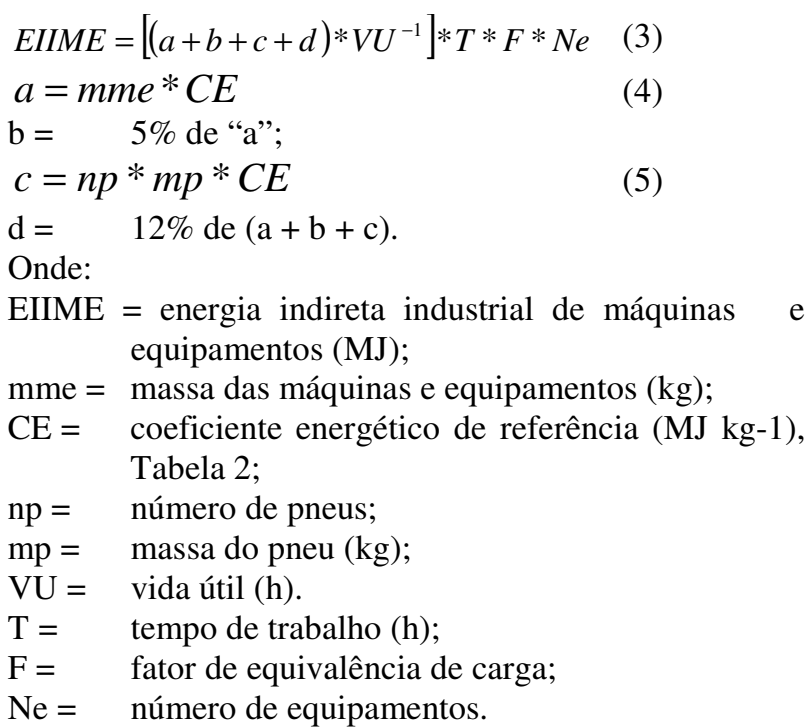

Tabela 02 - Coeficientes energéticos (MJ kg-1) correspondentes a cada tipo de material utilizado nas depreciações energéticas de máquinas ou equipamentos.

\begin{tabular}{lc}
\hline Máquinas e equipamentos & $\mathrm{CE}$ \\
\hline Alcooduto & 62,79 \\
Bombas booster do alcooduto & 62,79 \\
Válvula de bloqueio do alcooduto & 62,79 \\
\hline
\end{tabular}

Fonte: $\mathrm{O}$ autor.

\subsubsection{Energia do tipo indireta de fonte industrial sob a forma de depreciação de dutovias:}

A metodologia adotada para os cálculos foi a depreciação monetária considerando o valor residual igual a zero (BOVOLENTA, 2013), com adaptações. O valor monetário foi convertido em energia pela "intensidade energética", isto é, a energia consumida no Brasil em 2011 dividida pelo produto interno bruto em 2011. 
A energia consumida no Brasil em 2011 foi de 10,36 trilhões de MJ (BRASIL, 2012), enquanto que o produto interno bruto do Brasil em 2011 foi de 4,14 trilhões de Reais (IBGE, 2012). O percentual de energia consumida pelo setor de transporte em 2011 foi de, aproximadamente, $30 \%$ do total (BRASIL, 2012), enquanto que o percentual do PIB relacionado ao transporte em 2011 foi de, aproximadamente, 5\% do total (IBGE, 2012).

Assim, a depreciação da via, foi calculada da seguinte maneira:

O custo de implantação do trecho é de, aproximadamente, 1.500.000 US\$ $\mathrm{km}^{-1}$ (SÃO PAULO, 2012), multiplicado pela sua distância. O resultado dividiu-se pela sua vida útil, em anos de uso, que é de, aproximadamente, 40 anos (CERNE, 2000). Em seguida, este valor foi dividido pela quantidade média de veículos que trafegam por hora pelo trecho que é de 1 (um). Para finalizar a conversão, multiplicou-se pelo fator de equivalência de carga do modal e pela "intensidade energética" $\left(14,81 \mathrm{MJ} \mathrm{R} \$^{-1}\right)$.

A conversão foi feita pelas Equações 6 e 7:

$$
\begin{aligned}
& \left.E I I M=I E^{*} F *\left\{(C I T * D) * P M * V U^{-1}\right] * V h^{-1}\right\} \\
& I E=E C B * P I B^{-1}
\end{aligned}
$$

Onde:

EIIM = energia indireta industrial de manutenção (MJ);

$\mathrm{IE}=\quad$ intensidade Energética $\left(\mathrm{MJ} \mathrm{R}^{-1}\right)$;

$\mathrm{F}=$ fator de equivalência de carga;

$\mathrm{ECB}=$ energia consumida no Brasil em 2011 (MJ);

$\mathrm{PIB}=$ produto interno bruto do Brasil em $2011(\mathrm{R} \$) ;$

CIT $=$ custo de implantação do trecho

$\left(\mathrm{R} \$ \mathrm{~km}^{-1}\right)$;

$\mathrm{D}=$ distância do trecho $(\mathrm{km})$;

$\mathrm{PM}=$ percentual de manutenção;

$\mathrm{VU}=\quad$ vida útil $(\mathrm{h})$;

$\mathrm{Vh}=\quad$ veículos por hora;

\subsection{Indicadores de eficiência energética}

Foram construídos indicadores energéticos no sentido de mensurar e comparar relações e grandezas que "entram" e "saem" do sistema produtivo. Para a análise foram determinados os seguintes indicadores:

2.4.1 Balanço energético renovável comparativo às entradas de energias fósseis

A determinação foi feita através da Equação 8:

$$
B E r=\Sigma S E R T-\Sigma E E N R T
$$

Onde:

$\mathrm{BEr}=\quad$ balanço energético renovável $(\mathrm{MJ})$;

$\Sigma$ SERT = somatório da "saída" energética renovável total (MJ);

$\Sigma$ EENRT = somatório das "entradas" energéticas nãorenováveis totais $(\mathrm{MJ})$.

2.4.2 Balanço energético líquido comparativo às entradas de energias totais
A determinação foi feita através da Equação 9:

$$
B E l=\Sigma S E R T-\Sigma E E T
$$

Onde:

$\mathrm{BEl}=$ balanço energético líquido $(\mathrm{MJ})$;

$\Sigma \mathrm{SERT}=\quad$ somatório da "saída" energética renovável total $(\mathrm{MJ})$;

$\Sigma E E T=\quad$ somatório das "entradas" energéticas totais $(\mathrm{MJ})$.

\subsubsection{Eficiência energética renovável comparativa às entradas de energias fósseis}

A determinação foi feita através da Equação 10:

$$
E E r=\Sigma S E R T * \Sigma E E N R T^{-1}
$$

Onde:

$\mathrm{EEr}=$ eficiência energética renovável;

$\Sigma$ SERT $=\quad$ somatório da "saída" energética renovável total $(\mathrm{MJ})$;

$\Sigma E E N R T=\quad$ somatório das "entradas" energéticas não-renováveis totais $(\mathrm{MJ})$.

\subsubsection{Eficiência energética global comparativa às entradas de energias totais}

A determinação foi feita através da Equação 11:

$$
E E g=\Sigma S E R T * \Sigma E E T^{-1}
$$

Onde:

$\mathrm{EEg}=$ eficiência energética global;

$\Sigma$ SERT = somatório da "saída" energética renovável total (MJ);

$\Sigma E E T=$ somatório das "entradas" energéticas nãorenováveis totais $(\mathrm{MJ})$.

\section{RESULTADOS E DISCUSSÕES}

As entradas de energia para o $1^{\circ}$ e $2^{\circ}$ trecho desta rota estão apresentados nas Tabelas 3 e 4 , respectivamente considerando o fator de equivalência.

Analisando-se os dados do $1^{\circ}$ trecho, verifica-se uma maior participação de energia do tipo indireta $(99,91 \%)$, representada pela fonte industrial $(98,52 \%)$. Tal demanda é explicada pela grande quantidade de aço para a construção dos alcoodutos.

A participação de energia do tipo direta $(0,09 \%)$ foi baixa em relação à indireta, devido a dutovia não utilizar óleo diesel para a condução do etanol. 
Tabela 3 - Entrada de energia, por tipo, fonte e forma, em MJ, e participações percentuais na operação de transporte dutoviário R10 multimodal de Anhembí (SP) à Paulínia (SP)

\begin{tabular}{|c|c|c|}
\hline \multirow[t]{2}{*}{$\underline{\text { TIPO }}$, fonte e forma } & \multicolumn{2}{|c|}{ Entradas de energia } \\
\hline & (MJ) & (\%) \\
\hline ENERGIA DIRETA & $4.886,55$ & $\underline{\mathbf{0 , 0 9}}$ \\
\hline Elétrica & $3.982,77$ & $\overline{81,50}$ \\
\hline 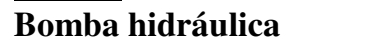 & $\mathbf{3 . 9 8 2 , 7 7}$ & $\overline{100,00}$ \\
\hline Biológica & $\underline{903,78}$ & $\underline{18,50}$ \\
\hline Mão-de-obra & 903,78 & 100,00 \\
\hline ENERGIA INDIRETA & $\underline{\mathbf{5 . 6 1 8 . 8 2 5 , 7 3}}$ & $\underline{99,91}$ \\
\hline Industrial & $\overline{5.535 .549,19}$ & $\overline{98,52}$ \\
\hline Alcooduto & $5.535 .549,19$ & 100,00 \\
\hline Industrial de depreciação & $\underline{83.276,54}$ & $\underline{1,48}$ \\
\hline Dutovia & $83.276,54$ & 100,00 \\
\hline SUBTOTAL & $5.623 .712,28$ & 100,00 \\
\hline
\end{tabular}

Fonte: $\mathrm{O}$ autor.

Analisando-se os dados do $2^{\circ}$ trecho, verifica-se uma maior participação de energia do tipo indireta $(99,81 \%)$, representada pela fonte industrial $(96,41 \%)$. Tal demanda é explicada pela grande quantidade de aço para a construção dos alcoodutos.
A participação de energia do tipo direta $(0,19 \%)$ foi baixa em relação à indireta, devido a dutovia não utilizar óleo diesel para a condução do etanol.

Tabela 4 - Entrada de energia, por tipo, fonte e forma, em MJ, e participações percentuais na operação de transporte dutoviário de Paulínia (SP) à São Sebastião (SP).

\begin{tabular}{lll}
\hline TIPO fonte e forma & \multicolumn{2}{c}{ Entradas de energia } \\
\cline { 2 - 3 } & $(\mathrm{MJ})$ & $(\%)$ \\
\hline ENERGIA DIRETA & $\frac{\mathbf{1 . 7 9 1 , 7 8}}{1.609,2}$ & $\underline{\mathbf{0 , 1 9}}$ \\
\hline Elétrica & $\mathbf{1 . 6 0 9 , 2}$ & $\underline{\mathbf{1 0 0 , 8 0}}$ \\
Bomba hidráulica & $\underline{182,58}$ & $\underline{10,19}$ \\
Biológica & $\underline{\mathbf{1 8 2 , 5 8}}$ & $\mathbf{1 0 0 , 0 0}$ \\
Mão-de-obra & $\underline{\mathbf{9 3 2 . 6 0 7 , 8 7}}$ & $\underline{\mathbf{9 9 , 8 1}}$ \\
ENERGIA INDIRETA & $\underline{89.129,86}$ & $\underline{\mathbf{8 9 9 , 4 1}}$ \\
\hline Industrial & $\underline{33.478,01}$ & $\underline{\mathbf{1 0 0 , 0 0}}$ \\
Alcooduto & $\mathbf{3 3 . 4 7 8 , 0 1}$ & $\underline{\mathbf{1 0 0 , 0 0}}$ \\
Industrial de depreciação & $\mathbf{9 3 4 . 3 9 9 , 6 5}$ & $\mathbf{1 0 0 , 0 0}$ \\
\hline Dutovia & &
\end{tabular}

Fonte: $\mathrm{O}$ autor.

Analisando-se o consumo específico de energia, verificase um resultado relativamente baixo, do ponto de vista energético, que corresponde a $0,14 \mathrm{MJ} \mathrm{km}-1 \mathrm{~m}-3$. Tal demanda é explicada pela ausência de entradas de energia fóssil e movimentar altos volumes de carga em relação aos outros modais.
Os Indicadores de balanço e eficiência energética, ou seja, o Ganho Energético líquido (GEl), a Eficiência Energética global (EEg), o Balanço Energético renovável (BEr) e a Eficiência Energética renovável (EEr) desta rota podem ser encontrados na tabela 5. Tais valores demonstram a enorme eficiência do sistema, no que se refere, às entradas e saídas energéticas.

Tabela 5 - Valor obtido a partir dos indicadores de balanço energético (em MJ) e eficiência energética (adimensional)

\begin{tabular}{lll} 
& $(\mathbf{M J})$ \\
\hline GEl & $1.585 .958 .977,00$ & \\
$\mathbf{E E g}$ & $1.593 .900 .000,00$ & 200,72 \\
$\mathbf{B E r}$ & & \\
$\mathbf{E E r}$ & & $-\cdots--$ \\
\hline
\end{tabular}

Fonte: O autor. 


\section{CONCLUSÃO}

A rota estudada, com $837 \mathrm{~km}$ percorridos, teve um único modal combinado em dois trechos, duto-dutoviário. $\mathrm{O}$ resultado obtido desta rota foi um consumo específico de energia de $0,14 \mathrm{MJ} \mathrm{km}^{-1} \mathrm{~m}^{-3}$. Este resultado foi muito menor em comparação aos resultados encontrados em (BOVOLENTA, 2013), onde a mesma rota só que, rodorodoviário, resultou em $0,53 \mathrm{MJ} \mathrm{km}^{-1} \mathrm{~m}^{-3}$ e a mesma rota ferro-feroviário, resultou em $0,27 \mathrm{MJ} \mathrm{km}^{-1} \mathrm{~m}^{-3}$. Quando foram contabilizados o Ganho Energético líquido (GEl), a Eficiência Energética global (EEg) e o Balanço Energético renovável (BEr), obteve-se respectivamente: 1.585.958.977,00 MJ; 200,72 e 1.593.900.000,00 MJ. A Eficiência Energética renovável (EEr) é igual "zero", ou seja, para se transportar etanol por dutos não se consome óleo diesel. Consome-se apenas energia elétrica das bombas hidráulicas.

O estudo feito neste trabalho gera uma reflexão: o modal dutoviário é o melhor meio de transporte para produtos a granel líquidos, mesmo sendo alto o valor de implantação de sua via em comparação as rodovias, ferrovias e hidrovias. Este tipo de estudo demonstra uma alternativa de escoamento de etanol por meio de dutos, que é mais sustentável energeticamente, ou seja, possibilita a utilização mais racional ou quase zero de combustível não renovável tal como o óleo diesel.

\section{REFFERENCIAS}

AINSWORTH, B. E. Compendium of physical activities: classification of energy costs of human physical activities. Medicine and Science in Sports and Exercise's, Indianápolis, v. 25, n. 1, p. 71-80, 1993.

Bovolenta, F. C; BIAGgiOni, M. A. M. Análise energética na Logística de transporte multimodal da soja. Revista Energia na Agricultura, Botucatu, v. 24, n. 3, p. 6, 2009.

BOVOLENTA, F. C. Análise energética na logística de transporte multimodal para exportação do etanol de Aparecida do Taboado (MS) ao porto de São Sebastião (SP). 2013. 133 f. Tese (Doutorado em Agronomia/Energia na Agricultura)-Faculdade de Ciências Agronômicas, Universidade Estadual Paulista, Botucatu, 2013.

BRASIL. Agência Nacional de Petróleo, Gás Natural e Biocombustíveis. Despacho do Superintendente $\mathbf{n}^{\mathbf{0}}$ 1.464, de 14 de setembro de 2010. Diário Oficial da União, Brasília, DF, 15 set. 2010.

BRASIL. Ministério de Minas e Energia. Balanço energético nacional - BEN - 2011. Disponível em: <http://www.mme.gov.br/EE33D585-275D-42C3-8013 556AB755C8CF/FinalDownload/DownloadIdD39F9F06B8F0009D9EAC82B64020A740/EE33D585275D-42C3-8013-

556AB755C8CF/mme/galerias/arquivos/publicacoes/BE
N/2_-_BEN_-_Ano_Base/1_-_BEN_Portugues__Inglxs_-_Completo.pdf>. Acesso em: 17 jul. 2012.

BRASIL. Centro Nacional das Indústrias do Setor Sucroenergético e Biocombustíveis. Consultoria em etanol e açúcar: Informações sobre exportação de etanol. Disponível em:

$<$ http://www.ceisebr.com/site/index.php/conteudo/visual izar/mercado-de-exportacao-para-o-etanol-sera-de-25-bide-litros>. Acesso em: 17 jul. 2012.

\section{CEISE - CENTRO NACIONAL DAS INDÚSTRIAS DO SETOR SUCROENERGÉTICO E BIOCOMBUSTÍVEIS. Consultoria em etanol e açúcar: informações sobre exportação de etanol. Disponível em: $<$ http://www.ceisebr.com/site/index.php/conteudo/visual izar/mercado-de-exportacao-para-o-etanol-sera-de-25-bi- de-litros>. Acesso em: 17 jul. 2012.}

\section{CERNE - CENTRO DE ESTUDOS EM RECURSOS NATURAIS E ENERGIA. Estudo de vida útil econômica e taxa de depreciação. Escola Federal de Engenharia de Itajubá, 2000. vol. 1/2.}

CONAB. Companhia Nacional de Abastecimento. Levantamentos de Safra. Disponível em: $<\mathrm{http} / / / \mathrm{www}$. conab.gov.br/conteudos.php?a=1253\&t=>. Acesso em: 9 mai. 2012.

IBGE. Produto interno bruto. Disponível em: $<$ http://www.ibge.gov.br/home/presidencia/noticias/notic ia_visualiza.php?id_noticia=1830\&id_pagina $=1>$. Acesso em: 17 jul. 2012.

LOGUM LOGÍSTICA S.A. Informações sobre dutos. Disponível em: <http://www.logum.com.br/php/faleconosco.php>. Acesso em: 19 ago. 2013.

MOREIRA, C. R. Avaliação energética do cultivo de eucalipto, com e sem composto de lixo urbano.

Revista Energia na Agricultura, Botucatu, v. 20, n. 4, p. 1-19, 2005.

UNIÃO DA INDÚSTRIA DE CANA-DE-AÇÚCAR UNICA. Regiões cultiváveis no Brasil. Disponível em: $<$ http://www.unica.com.br/content/default.asp?cchCode= \{C2B8C535-736F-406B-BEB2-5D12B834EF59\}>. Acesso em: 17 jul. 2012.

\section{UNIDUTO LOGÍSTICA S.A. Informações sobre}

dutos. Disponível em:

$<$ http://www.uniduto.com.br/perfil.php?menu=1\&item= 1>. Acesso em: 26 jul. 2012. Fazer citação correta no texto 